\title{
Optimized Schwarz methods with overlap for the Helmholtz equation
}

Martin J. Gander and Hui Zhang

\section{Introduction}

For the Helmholtz equation, simple absorbing conditions of the form $\partial_{n}-i \omega$ were proposed as transmission condition (TC) in Schwarz methods first without overlap in [4], and later also with overlap, see [3, 12]. More advanced TCs can also be used, see e.g. [11, 14, 2]. Furthermore, parameters can be introduced into TCs and then optimized for rapid convergence, which led to the so called optimized Schwarz methods, see e.g. [6, 13] for elliptic equations. Without overlap, the parameters involved in some zero- and second-order TCs for the Helmholtz equation have been optimized in [8,7]. With overlap, preliminary numerical studies of the parameters have been presented in $[5,9]$. In this paper, we present the asymptotic solutions of the corresponding optimization problems with small overlap. We also compare the optimized parameters with other choices based on convergence factors and actual iteration numbers. We finally test for the first time Taylor second-order absorbing conditions for domain decomposition with overlap in the Helmholtz case.

\section{Schwarz Methods with Overlap}

As a model problem, we consider the Helmholtz equation in free space,

$$
\left(\omega^{2}+\Delta\right) u=f(x, y), \quad(x, y) \in \mathbb{R} \times \mathbb{R}^{d-1},
$$

equipped with the Sommerfeld radiation condition

$$
\lim _{r \rightarrow \infty} r^{\frac{d-1}{2}}\left(\frac{\partial u}{\partial r}-i \omega\right)=0, \quad r=\sqrt{x^{2}+\sum_{j=1}^{d-1} y_{j}^{2}}
$$

We decompose the domain into two overlapping subdomains $\Omega_{1}=(-\infty, L) \times \mathbb{R}^{d-1}$ and $\Omega_{2}=(0, \infty) \times \mathbb{R}^{d-1}$ with the overlap size $L>0$. The Schwarz iteration reads

$$
\begin{aligned}
\omega^{2} u_{1}^{n+1}+\Delta u_{1}^{n+1} & =f(x, y), & (x, y) \in \Omega_{1}, \\
\left(\partial_{x}+\mathscr{S}_{1}\right)\left(u_{1}^{n+1}\right)(L, y) & =\left(\partial_{x}+\mathscr{S}_{1}\right)\left(u_{2}^{n}\right)(L, y), & y \in \mathbb{R}^{d-1},
\end{aligned}
$$

Section of Mathematics, University of Geneva, 2-4 rue du Liévre, Case postale 64, \{martin.gander, hui.zhang\}@unige.ch 
and

$$
\begin{aligned}
\omega^{2} u_{2}^{n+1}+\Delta u_{2}^{n+1} & =f(x, y), & (x, y) \in \Omega_{2}, \\
\left(-\partial_{x}+\mathscr{S}_{2}\right)\left(u_{2}^{n+1}\right)(0, y) & =\left(-\partial_{x}+\mathscr{S}_{2}\right)\left(u_{1}^{n}\right)(0, y), & y \in \mathbb{R}^{d-1}
\end{aligned}
$$

where $\mathscr{S}_{j}, j=1,2$ are two linear operators in some trace spaces along $\{L\} \times \mathbb{R}^{d-1}$ and $\{0\} \times \mathbb{R}^{d-1}$, respectively. For the analysis it suffices to consider by linearity the case $f(x, y)=0$ and to analyze convergence to the zero solution. We take a Fourier transform in the $y$ direction to obtain

$$
\begin{aligned}
\left(\omega^{2}-|k|^{2}\right) \hat{u}_{1}^{n+1}+\partial_{x x}^{2} \hat{u}_{1}^{n+1} & =0, \quad x \in(-\infty, L), \\
\left(\partial_{x}+s_{1}\right)\left(\hat{u}_{1}^{n+1}\right)(L, k) & =\left(\partial_{x}+s_{1}\right)\left(\hat{u}_{2}^{n}\right)(L, k),
\end{aligned}
$$

and

$$
\begin{aligned}
\left(\omega^{2}-|k|^{2}\right) \hat{u}_{2}^{n+1}+\partial_{x x}^{2} \hat{u}_{2}^{n+1} & =0, \quad x \in(0, \infty), \\
\left(-\partial_{x}+s_{2}\right)\left(\hat{u}_{2}^{n+1}\right)(0, k) & =\left(-\partial_{x}+s_{2}\right)\left(\hat{u}_{1}^{n}\right)(0, k),
\end{aligned}
$$

where $k$ is the Fourier variable of $y$ and $s_{j}$ denotes the symbol of $\mathscr{S}_{j}$. Since the Sommerfeld radiation condition excludes growing solutions as well as incoming modes at infinity we obtain the solutions

$$
\begin{aligned}
& \hat{u}_{1}^{n+1}(x, k)=\hat{u}_{1}^{n+1}(L, k) e^{\lambda(k)(x-L)}, \\
& \hat{u}_{2}^{n+1}(x, k)=\hat{u}_{2}^{n+1}(0, k) e^{-\lambda(k) x},
\end{aligned}
$$

where $\lambda(k)$ denotes the root of the characteristic equation $\lambda^{2}+\left(\omega^{2}-|k|^{2}\right)=0$ with positive real part or negative imaginary part,

$$
\lambda(k):= \begin{cases}\sqrt{|k|^{2}-\omega^{2}} & \text { for }|k|>\omega, \\ -i \sqrt{\omega^{2}-|k|^{2}} & \text { for }|k|<\omega .\end{cases}
$$

Substitution of the solutions into the transmission conditions yields

$$
\begin{aligned}
& \hat{u}_{1}^{n+1}(L, k)=\frac{s_{1}(k)-\lambda(k)}{s_{1}(k)+\lambda(k)} e^{-\lambda(k) L} \hat{u}_{2}^{n}(0, k), \\
& \hat{u}_{2}^{n+1}(0, k)=\frac{s_{2}(k)-\lambda(k)}{s_{2}(k)+\lambda(k)} e^{-\lambda(k) L} \hat{u}_{1}^{n}(L, k) .
\end{aligned}
$$

By recursion we have $\hat{u}_{1}^{n+1}(L, k)=\rho(k) \hat{u}_{1}^{n-1}(L, k)$ and $\hat{u}_{2}^{n+1}(0, k)=\rho(k) \hat{u}_{2}^{n-1}(0, k)$, where the convergence factor $\rho$ for a double iteration is defined by

$$
\rho(k)=\frac{s_{1}(k)-\lambda(k)}{s_{1}(k)+\lambda(k)} \cdot \frac{s_{2}(k)-\lambda(k)}{s_{2}(k)+\lambda(k)} e^{-2 \lambda(k) L} .
$$

Setting the two complex parameters $s_{1}=p_{1}-i q_{1}$ and $s_{2}=p_{2}-i q_{2}$, with $p_{j}, q_{j} \in \mathbb{R}$, and inserting $s_{1}, s_{2}$ and (1) into the convergence factor (2), we find after simplifying 
$\left|\rho\left(p_{1}, q_{1}, p_{2}, q_{2}, k\right)\right|^{2}= \begin{cases}\frac{p_{1}^{2}+\left(q_{1}-\sqrt{\omega^{2}-|k|^{2}}\right)^{2}}{p_{1}^{2}+\left(q_{1}+\sqrt{\omega^{2}-|k|^{2}}\right)^{2}} \frac{p_{2}^{2}+\left(q_{2}-\sqrt{\omega^{2}-|k|^{2}}\right)^{2}}{p_{2}^{2}+\left(q_{2}+\sqrt{\omega^{2}-|k|^{2}}\right)^{2}}, & |k|^{2}<\omega^{2}, \\ \frac{q_{1}^{2}+\left(p_{1}-\sqrt{|k|^{2}-\omega^{2}}\right)^{2}}{q_{1}^{2}+\left(p_{1}+\sqrt{|k|^{2}-\omega^{2}}\right)^{2}} \frac{q_{2}^{2}+\left(p_{2}-\sqrt{|k|^{2}-\omega^{2}}\right)^{2}}{q_{2}^{2}+\left(p_{2}+\sqrt{|k|^{2}-\omega^{2}}\right)^{2}} e^{-4 \lambda(k) L}, & |k|^{2}>\omega^{2} .\end{cases}$

As long as $|k| \neq \omega$ and $p_{j}, q_{j}>0$, we have $|\rho|<1$.

Remark 1. It was shown in [6] that the two-sided operators $\mathscr{S}_{j}=s_{j} \in \mathbb{C}$ can be transformed into the second-order operators

$$
\tilde{\mathscr{S}}_{1}=\tilde{\mathscr{S}}_{2}=r_{1}-r_{2} \nabla_{y}^{2}, \quad \text { with } \quad r_{1}=\frac{-\omega^{2}+s_{1} s_{2}}{s_{1}+s_{2}}, \quad r_{2}=\frac{1}{s_{1}+s_{2}},
$$

and the associated convergence factor for a single iteration is then given by

$$
\tilde{\rho}(k)=\frac{s_{1}(k)-\lambda(k)}{s_{1}(k)+\lambda(k)} \cdot \frac{s_{2}(k)-\lambda(k)}{s_{2}(k)+\lambda(k)} e^{-\lambda(k) L},
$$

which is just (2) with $L$ replaced by $L / 2$.

\section{Optimized transmission conditions}

For simplicity, we consider $p_{1}=q_{1}, p_{2}=q_{2}$. Our goal is to find good parameters $p_{1}, p_{2}$ such that the modulus of the squared convergence factor (3) is as small as possible over a range of frequencies $|k| \in\left[k_{\min }, k_{-}\right] \cup\left[k_{+}, k_{\max }\right]$, where $k_{-}<\omega<k_{+}$. We require $|k|$ to be away from $\omega$ because $|\rho|=1$ when $|k|=\omega$, independently of what one chooses for the parameters $p_{j}$ and $q_{j}$. Since in general we do not know how the Fourier coefficients of the initial error are distributed over the frequencies, we minimize $|\rho|$ for the worst case, that is, we solve the min-max problem

$$
\operatorname{argmin}_{\left(p_{1}, p_{2}\right) \in \mathbb{P}}\left(\max _{|k| \in\left[k_{\min }, k_{-}\right] \cup\left[k_{+}, k_{\max }\right]}\left|\rho\left(p_{1}, p_{1}, p_{2}, p_{2}, k\right)\right|^{2}\right),
$$

where $\mathbb{P}$ is a certain search domain of the parameters. For well-posedness of the subdomain problems, we should choose $\mathbb{P} \subset[0, \infty)^{2}$. The best approximation problem (6) is difficult to solve, and we only give asymptotic formulas for the parameters such that the convergence factor is as small as possible in different limiting processes in the mesh size $h$ and the wave number $\omega$.

The proofs of the following theorems are beyond the scope of this short paper and will appear in [10].

Theorem 1. Let $L=C_{L} h, k_{\max } \in[C / h, \infty], C_{L}, C, k_{\min }, k_{-}, k_{+}$and $\omega$ be positive and independent of $h, k_{\min }<k_{-}<\omega, k_{\max }>k_{+}>\omega$ and $\mathbb{P}=\left\{\left(p_{1}, p_{2}\right) \mid 0 \leq p_{1} \leq p_{2}<\right.$ $\infty\}$. Suppose $h$ is small and $|k| \in\left[k_{\min }, k_{-}\right] \cup\left[k_{+}, k_{\max }\right]$. If we set 


$$
\begin{aligned}
& p_{1}=p_{1}^{*}=C_{\omega}^{2 / 5}(4 L)^{-1 / 5} / 2+o\left(h^{-1 / 5}\right) \\
& p_{2}=p_{2}^{*}=C_{\omega}^{1 / 5}(4 L)^{-3 / 5}+o\left(h^{-3 / 5}\right)
\end{aligned}
$$

where $C_{\omega}=\min \left\{\omega^{2}-k_{-}^{2}, k_{+}^{2}-\omega^{2}\right\}$, then (3) is bounded by $1-4\left(4 L \sqrt{C_{\omega}}\right)^{1 / 5}+$ $o\left(h^{1 / 5}\right)$. Moreover, any solution of (6) must satisfy (7).

Theorem 2. Let $L=C_{L} h, h=C_{h} / \omega^{\gamma}, \gamma \geq 1, k_{\max } \in[C / h, \infty], \delta_{\omega}=\min \{\omega-$ $\left.k_{-}, k_{+}-\omega\right\}$ with $C_{L}, C_{h}, C, k_{-}$and $k_{+}$positive constants independent of $\omega, k_{\min }<$ $k_{-}<\omega, k_{\max }>k_{+}>\omega$ and $\mathbb{P}=\left\{\left(p_{1}, p_{2}\right) \mid 0 \leq p_{1} \leq p_{2}<\infty\right\}$. Suppose $\omega$ is large and $|k| \in\left[k_{\min }, k_{-}\right] \cup\left[k_{+}, k_{\max }\right]$. Then, for $1 \leq \gamma<9 / 8$ any solution of $(6)$ satisfies

$$
\begin{aligned}
& p_{1}^{*}=\delta_{\omega}^{3 / 8}(\omega / 2)^{5 / 8}+o\left(\omega^{5 / 8}\right), \\
& p_{2}^{*}=\left(2 \delta_{\omega}\right)^{1 / 8} \omega^{7 / 8}+o\left(\omega^{7 / 8}\right),
\end{aligned}
$$

for which (3) is bounded by $1-4 \cdot 2^{1 / 8} \delta_{\omega}^{1 / 8} \omega^{-1 / 8}+o\left(\omega^{-1 / 8}\right)$. For $\gamma>9 / 8$, any solution of (6) satisfies

$$
\begin{aligned}
& p_{1}^{*}=\left(\delta_{\omega} \omega\right)^{2 / 5} L^{-1 / 5} / 2+o\left(\omega^{2 / 5+\gamma / 5}\right), \\
& p_{2}^{*}=\left(\delta_{\omega} \omega\right)^{1 / 5} L^{-3 / 5} / 2+o\left(\omega^{1 / 5+3 \gamma / 5}\right),
\end{aligned}
$$

and (3) is bounded by $1-4 \sqrt{2}\left(C_{h} C_{L}\right)^{1 / 5} \delta_{\omega}^{1 / 10} \omega^{1 / 10-\gamma / 5}+o\left(\omega^{1 / 10-\gamma / 5}\right)$. Finally, for $\gamma=9 / 8$, any solution of (6) satisfies

$$
\begin{aligned}
& p_{1}^{*}=\left(C_{h} C_{L} \delta_{\omega}\right)^{1 / 3} \omega^{5 / 8}+o\left(\omega^{5 / 8}\right), \\
& p_{2}^{*}=C_{h} C_{L} \omega^{7 / 8}+o\left(\omega^{7 / 8}\right),
\end{aligned}
$$

and (3) is bounded by

$$
\begin{cases}1-16 C_{h} C_{L} \omega^{-1 / 8}+o\left(\omega^{-1 / 8}\right), & \text { if } 2^{-15 / 8} \delta_{\omega}^{1 / 8} \leq C_{h} C_{L}, \\ 1-2 \sqrt{2} \delta_{\omega}^{1 / 6} C_{h}^{-1 / 3} C_{L}^{-1 / 3} \omega^{-1 / 8}+o\left(\omega^{-1 / 8}\right), & \text { if } 2^{-15 / 8} \delta_{\omega}^{1 / 8} \geq C_{h} C_{L} .\end{cases}
$$

Remark 2. In the particular case $\gamma=9 / 8$, the constant in front of the leading term of $p_{1}^{*}$ can be an arbitrary number in the interval $\left[\sqrt{2 \delta_{\omega}} /\left(8 C_{h} C_{L}\right), 32 C_{h}^{3} C_{L}^{3}\right]$ in order to solve (6). But the choice in the above theorem is the best in the sense that it simultaneously minimizes the maximum of the other local but not global maxima.

Remark 3. In practice, we use only the leading order terms of the optimized parameters. But it is also possible to extract higher order terms.

Fig.1 shows the convergence factors of different Schwarz methods, obtained for the model problem in $\mathbb{R}^{2}$, with $\omega=20 \pi$ and $h=1 / 100$. The maximum of the convergence factors for double iterations over the chosen interval $k \in[\pi, \omega-\pi] \cup[\omega+$ $\pi, \pi / h]$ are 1.0, for the classical Schwarz method and Després' method without overlap [4], 0.4376 for Després' method with overlap [3, 12], 0.1548 for the optimized Schwarz methods without overlap [7], and and 0.0764 for the same method with 


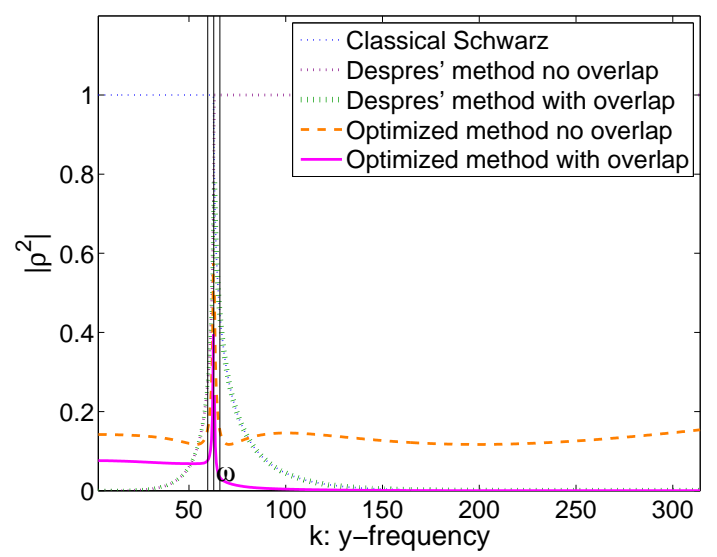

Fig. 1 Convergence factors of different Schwarz methods as functions of the Fourier parameter $k$, for $\omega=20 \pi, h=1 / 100$. The vertical lines indicate the $\omega, \omega-\pi$ and $\omega+\pi$ which are used to exclude a short interval for the optimized methods.

overlap. The overlap size we chose is $2 h$, and we used the second-order formulation (4), (5) for the optimized methods.

\section{Numerical experiments}

We used the ORAS formulation described in [13] for our implementation. As an alternative, one could also use a substructured formulation, see e.g. [9]. We implemented the second-order transmission conditions as indicated in Remark 1. We always solve the homogeneous equation with the zero solution and use a random initial guess to stimulate all frequencies. We use the domain decomposition $\Omega_{1}=\left(0, \frac{1}{2}+h\right) \times(0,1), \Omega_{2}=\left(\frac{1}{2}-h, 1\right) \times(0,1)$, and iterate until the relative residual is less than $10^{-8}$. We compare the overlapping Schwarz methods with optimized second-order transmission condition denoted by $\mathrm{OO} 2$ to those with the classical Dirichlet condition denoted by $\mathrm{Cl}$, simple absorbing conditions of the form $\partial_{n}-i \omega$ (i.e. Després' method with overlap, c.f. $[3,12])$ denoted by TO0, because it corresponds to a Taylor expansion of zero order of the symbol of the DtN operator, and the second-order low frequency absorbing condition, which is denoted by TO2. Since the Schwarz methods can be used as a stationary iterative solver, or as a preconditioner for GMRES, both cases are tested, except for the classical Schwarz stationary iteration, which can not converge.

We consider the open cavity problem with homogeneous Dirichlet boundary conditions on the top and the bottom of the unit square and the TO2 second-order absorbing conditions [1] on the left and the right sides, and also the free space problem 
Table 1 Iteration numbers for the open cavity problem on the left and for the free space problem on the right, top half for $\omega=9.5 \pi$, and below for $\omega=10 \pi$.

\begin{tabular}{|c|c|c|c|c|c|c|c|c|c|c|c|c|c|c|}
\hline \multirow[t]{2}{*}{$1 / h$} & \multicolumn{3}{|c|}{ Stationary } & \multicolumn{4}{|c|}{ GMRES } & \multicolumn{3}{|c|}{ Stationary } & \multicolumn{4}{|c|}{ GMRES } \\
\hline & $\overline{\mathrm{TO}}$ & TO2 & $\overline{\mathrm{OO} 2}$ & Cl. & TO0 & TO2 & $\mathrm{OO} 2$ & TO0 & TO2 & $\overline{\mathrm{OO} 2}$ & $\mathrm{Cl}$. & TO0 & TO2 & $\mathrm{OO} 2$ \\
\hline 50 & 34 & 35 & 14 & $\overline{25}$ & 16 & 15 & 12 & 23 & 24 & 17 & 25 & 17 & 15 & 13 \\
\hline 100 & 74 & 84 & 17 & 30 & 22 & 22 & 13 & 33 & 41 & 21 & 28 & 21 & 21 & 14 \\
\hline 200 & 166 & 172 & 20 & 38 & 27 & 32 & 14 & 51 & 73 & 22 & 33 & 27 & 30 & 15 \\
\hline 400 & 343 & 345 & 20 & 49 & 33 & 41 & 14 & 85 & 135 & 23 & 42 & 33 & 40 & 15 \\
\hline 800 & 662 & 717 & 21 & 67 & 40 & 50 & 16 & 144 & 249 & 24 & 58 & 42 & 49 & 16 \\
\hline 50 & 67 & 70 & $\overline{19}$ & 26 & 15 & 14 & $\overline{14}$ & 22 & 23 & 17 & 26 & 16 & 15 & $\overline{13}$ \\
\hline 100 & 227 & 222 & 31 & 30 & 21 & 22 & 15 & 32 & 40 & 20 & 27 & 21 & 21 & 14 \\
\hline 200 & 469 & 371 & 44 & 38 & 28 & 32 & 15 & 50 & 71 & 22 & 33 & 27 & 30 & 15 \\
\hline 400 & 681 & 455 & 51 & 51 & 34 & 42 & 15 & 83 & 130 & 22 & 43 & 34 & 40 & 15 \\
\hline 800 & 864 & 504 & 55 & 68 & 41 & 52 & 17 & 136 & 241 & 23 & 55 & 42 & 49 & 15 \\
\hline
\end{tabular}

truncated to the unit square with the TO2 second-order absorbing conditions at the boundary.

First, we fix $\omega=9.5 \pi$ (or $\omega=10 \pi$ ) which are away from (or on) the sine frequencies at the continuous level in the $y$-direction. The corresponding iteration numbers are listed in Table 1 . We can see that the minimum distance from $\omega$ to the frequencies at the discrete level in the $y$-direction plays an important role in all the stationary iterations while in the GMRES iterations this effect is only moderate. Fig. 2 shows the asymptotic behavior of the different Schwarz methods as $h \rightarrow 0$, for the open cavity problem, and confirms our Fourier analysis results in Theorem 1.

Now we fix $h \omega$ or $h \omega^{3 / 2}$ constant to see how the Schwarz methods behave for higher and higher wave numbers, which corresponds to Theorem 2. The iteration numbers are listed in Table 2. We see that the optimized method still converges faster than the others when used as a preconditioner for GMRES, while the stationary

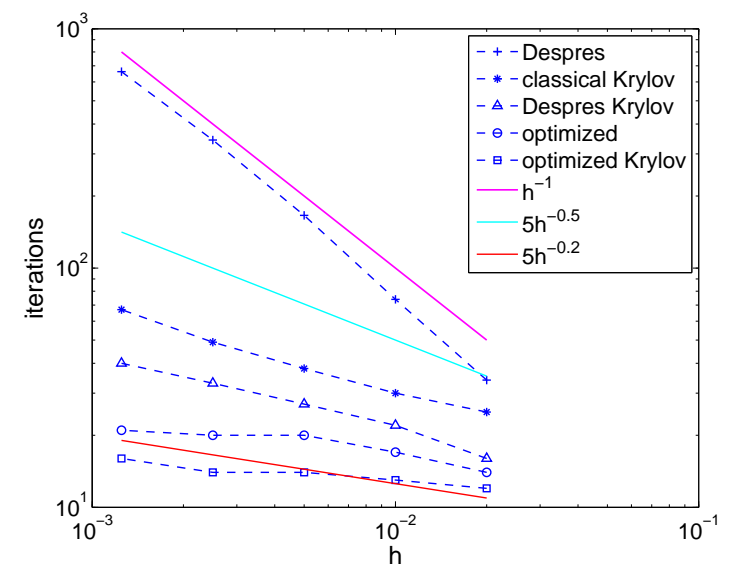

Fig. 2 Asymptotic behavior of the Schwarz methods for the open cavity, $\omega=9.5 \pi$. 
Table 2 Iteration numbers for the open cavity problem on the left and for the free space problem on the right, top half for $h \omega=\pi / 5$, and below for $h \omega^{3 / 2} \approx 3.52$.

\begin{tabular}{|c|c|c|c|c|c|c|c|c|c|c|c|c|c|c|}
\hline \multirow[t]{2}{*}{$1 / h$} & \multicolumn{3}{|c|}{ Stationary } & \multicolumn{4}{|c|}{ GMRES } & \multicolumn{3}{|c|}{ Stationary } & \multicolumn{4}{|c|}{ GMRES } \\
\hline & $\overline{\mathrm{TO} 0}$ & TO2 & $\overline{\mathrm{OO} 2}$ & $\mathrm{Cl}$. & TO0 & TO2 & $\overline{\mathrm{OO} 2}$ & $\overline{\mathrm{TO} 0}$ & TO2 & $\overline{\mathrm{OO} 2}$ & $\overline{\mathrm{Cl}}$. & TO0 & TO2 & $\overline{\mathrm{OO} 2}$ \\
\hline 100 & 86 & 67 & 35 & 38 & 20 & 19 & 16 & 27 & 27 & 18 & $\overline{35}$ & 20 & 18 & 15 \\
\hline 200 & - & 110 & - & 48 & 25 & 22 & 19 & 33 & 30 & 19 & 43 & 25 & 21 & 16 \\
\hline 400 & 280 & 150 & 72 & 69 & 38 & 32 & 20 & 43 & 37 & 19 & 53 & 28 & 25 & 17 \\
\hline 800 & 178 & 139 & 44 & 76 & 42 & 35 & 25 & 56 & 45 & 19 & 65 & 32 & 30 & 17 \\
\hline 100 & 80 & 87 & 15 & 34 & 22 & 20 & 14 & 29 & 31 & 19 & 30 & 21 & 18 & 14 \\
\hline 200 & - & 2948 & - & 43 & 27 & 27 & 19 & 42 & 39 & 19 & 34 & 27 & 24 & 15 \\
\hline 400 & 266 & 279 & 26 & 49 & 33 & 32 & 18 & 56 & 50 & 20 & 41 & 35 & 30 & 16 \\
\hline 800 & 208 & 218 & 21 & 70 & 46 & 41 & 18 & 78 & 65 & 20 & 47 & 43 & 37 & 16 \\
\hline
\end{tabular}

iterations are again greatly affected by the discrete frequencies close to the wave number. The bars in the tables represent divergence.

Next, we test the various Schwarz methods for an increasing number of subdomains. Since in most cases the stationary iterations diverge, we only show the GMRES iteration numbers in Table 3, where we use a bar to represent iteration numbers larger than 3000 . We can see, neglecting the numbers in the parentheses for the moment, that all the methods deteriorate rapidly and the overlapping TO2 method outperforms the others eventually. Clearly the optimization of the two subdomain convergence factor does not predict well the optimal choice in the case of many subdomains for the Helmholtz equation.

To partially improve the $\mathrm{OO} 2$ method, we introduce now two heuristics. First, we take $\delta_{\omega}=N \pi / 2$ instead of $\delta_{\omega}=\pi$ in the former experiments, where $N$ denotes the number of subdomains in the $x$-direction. Second, since the real parts of the parameters slow down the convergence for propagating modes, which becomes worse when the number of subdomains increases, we use $s_{j}=(2 / N-i) p_{j}(j=1,2)$ instead of $s_{j}=(1-i) p_{j}$. The new results are shown in the parentheses of Table 3 , where the first numbers are obtained by using the two heuristics and the second numbers are from numerically optimized parameters based on a new many-subdomain Fourier analysis. But still, the low frequency Taylor conditions perform best in these exper-

Table 3 Iteration numbers of GMRES, $h=1 / 256, \omega=51.2 \pi$, overlap $2 h$.

\begin{tabular}{|c|c|c|c|c|c|c|c|c|c|c|c|c|}
\hline \multirow[t]{2}{*}{ Sub. } & \multicolumn{6}{|c|}{ open cavity } & \multicolumn{6}{|c|}{ free space } \\
\hline & $\mathrm{Cl}$. & TOO & TO2 & & $\mathrm{OO} 2$ & & Cl. & TO0 & TO2 & & $\mathrm{OO} 2$ & \\
\hline $2 \times 1$ & 52 & 28 & 24 & 18 & & & 48 & 25 & 23 & 16 & & \\
\hline $4 \times 1$ & 396 & 68 & 46 & 68 & $(45$ & 40) & 163 & 29 & 24 & 45 & $(30$ & 22) \\
\hline $8 \times 1$ & - & 160 & 102 & 162 & (91 & 88) & - & 44 & 33 & 108 & $(50$ & 36) \\
\hline $16 \times 1$ & - & 682 & 221 & 492 & (183 & 188) & - & 88 & 65 & 258 & $(82$ & 67) \\
\hline $2 \times 2$ & 118 & 66 & 63 & 61 & & & 49 & 27 & 25 & 20 & & \\
\hline $4 \times 4$ & 2192 & 184 & 172 & 183 & $(177$ & 166) & 372 & 38 & 33 & 49 & $(42$ & $35)$ \\
\hline $8 \times 8$ & - & 789 & 618 & 734 & $(638$ & 601) & - & 69 & 65 & 104 & $(82$ & 70) \\
\hline $16 \times 16$ & - & 2047 & 1473 & 2268 & (1859 & 1514) & - & 123 & 127 & 184 & (168 & 136) \\
\hline
\end{tabular}


iments. Our on-going work is to take a closer look at the multi-domain case and to seek better choices of parameters if it is possible.

Acknowledgements The work was supported by the University of Geneva. The second author was also partially supported by the International Science and Technology Cooperation Program of China (2010DFA14700).

\section{References}

1. Bamberger, A., Joly, P., Roberts, J.E.: Second-order absorbing boundary conditions for the wave equation: a solution for the corner problem. SIAM J. Numer. Anal. 27, 323-352 (1990)

2. Boubendir, Y., Antoine, X., Geuzaine, C.: A quasi-optimal non-overlapping domain decomposition algorithm for the Helmholtz equation. J. Comput. Phys. 231, 262-280 (2012)

3. Cai, X.C., Casarin, M.A., Elliott Jr., F.W., Widlund, O.B.: Overlapping Schwarz algorithms for solving Helmholtz's equation. In: J. Mandel, C. Farhat, X.C. Cai (eds.) Domain Decomposition Methods 10, Contemporary Mathematics 218, Boulder, pp. 437-445. AMS (1998)

4. Després, B.: Domain decomposition method and the Helmholtz problem. In: G.C. Cohen, L. Halpern, P. Joly (eds.) Mathematical and numerical aspects of wave propagation phenomena, Strasbourg, pp. 44-52. SIAM (1991)

5. Gander, M.J.: Optimized Schwarz methods for Helmholtz problems. In: N. Debit, M. Garbey, R.H.W. Hoppe, et. al. (eds.) Domain Decomposition Methods in Science and Engineering, 13th International Conference on Domain Decomposition Methods, Barcelona, pp. 247-254. CIMNE (2002)

6. Gander, M.J.: Optimized Schwarz methods. SIAM J. Numer. Anal. 44, 699-731 (2006)

7. Gander, M.J., Halpern, L., Magoulès, F.: An optimized Schwarz method with two-sided Robin transmission conditions for the Helmholtz equation. Int. J. Numer. Meth. Fluids 55, 163-175 (2007)

8. Gander, M.J., Magoulès, F., Nataf, F.: Optimized Schwarz methods without overlap for the Helmholtz equation. SIAM J. Sci. Comput. 24, 38-60 (2002)

9. Gander, M.J., Zhang, H.: Domain decomposition methods for the Helmholtz equation: a numerical investigation. In: R. Bank, M. Holst, J. Xu (eds.) Domain Decomposition Methods in Science and Engineering XX, Lecture Notes in Computational Science and Engineering, San Diego, p. to appear. Springer-Verlag (2012)

10. Gander, M.J., Zhang, H.: Optimized Schwarz methods with overlap for the Helmholtz equation. in preparation (2012)

11. Ghanemi, S.: A domain decomposition method for Helmholtz scattering problems. In: P.E. Bjorstad, M.S. Espedal, D.E. Keyes (eds.) Proceedings of the 9th Intl. Conf. on Domain Decomposition Methods, Ullensvang, pp. 105-112. ddm.org (1998)

12. Kimn, J.H., Sarkis, M.: Restricted overlapping balancing domain decomposition methods and restricted coarse problems for the Helmholtz problem. Comput. Methods Appl. Mech. Engrg. 196, 1507-1514 (2007)

13. St-Cyr, A., Gander, M.J., Thomas, S.J.: Optimized multiplicative, additive, and restricted additive Schwarz preconditioning. SIAM J. Sci. Comput. 29, 2402-2425 (2007)

14. Stupfel, B.: Improved transmission conditions for a one-dimensional domain decomposition method applied to the solution of the Helmholtz equation. J. Comput. Phys. 229, 851-874 (2010) 